\title{
HYBRID NOISE FILTERING ALGORITHM BASED ON NEURO-TYPE 2 FUZZY SYSTEMS
}

\author{
ANKUSH RAI*, JAGADEESH KANNAN R
}

School of Computing Science \& Engineering, VIT University, Chennai, Tamil Nadu, India. Email: ankushressci@gmail.com

\author{
Received: 13 December 2016, Revised and Accepted: 03 April 2017
}

\begin{abstract}
Objetcive: Medical images are often subjected to noise due to the failure of data acquisition hardware at the source. Thus, making it difficult for the radiologist to perform image analysis and give correct diagnosis of the disease.
\end{abstract}

Methods: This research presents a new image denoising algorithm based on the combination of neuro-type 2 fuzzy systems. The method not only preserves the information relevant for diagnostic details but also provides a cost-effective solution for recovery of lost information due to noise.

Resulst and Conclusion: The pixel transformation achieved by the proposed method is found to be more efficient than the wavelet methods in image denoising particularly for the removal of Gaussian noise while ensuring that the denoising is done while keeping intact of the integrity and granularity of the image.

Keywords: Fuzzy-neural logic, Denoising image, Medical application.

(C) 2017 The Authors. Published by Innovare Academic Sciences Pvt Ltd. This is an open access article under the CC BY license (http://creativecommons. org/licenses/by/4. 0/) DOI: http://dx.doi.org/10.22159/ajpcr.2017.v10s1.19647

\section{INTRODUCTION}

With the board utilization of advanced imaging in medicine applications, the nature of computerized medicinal images has turned into a critical research topic. To remove Noise from the pictures is difficult. To accomplish the most ideal noise removal analyses, it is imperative for restorative pictures to be sharp, clear, and free of noise. Removal of noise in these digital pictures stays as one of the significant difficulties in the investigation of restorative imaging. In our exploration, we propose a powerful computation method for removing and restoring the medicinal pictures utilizing window based multiwavelet change.

A traditional crisis in image processing is noise reduction [1]. Digital image reconstruction is a robust means by which the underlying images masked in blurry and noisy data can be uncovered. Its input data are sensitive to noise measurement, which can be magnified firmly, resulting in huge artefacts in the reconstructed image. By reconstructing the permitted images [2], this intricacy can be eliminated. As it can be analyze with only a few corrupted pixels which quite significant for noise removal. However, if we lose a particular area in case of medical images, then it will turn out to be grave scenario. Therefore, denoising of medical images is further essential which leads physician for precise analysis of diseases. Medical images such as X-ray, computed tomography (CT), magnetic resonance imaging (MRI), positron emission tomography, and single photon emission CT encompass diminutive information about human organs. For determining the internal structure of an object, X-ray CT is an effective method for regional application, such as in the nondestructive testing for huge sets of materials. It allows data acquisition from a varied angle and observation viewpoints, the CT image is derived, and with the support of a computer (Radon transform) the final reconstructed CT image produced. It is deplorable for a human being from adversaries, when these medical images are corrupted by noise. In both image processing and biomedical engineering, CT image denoising is an important research topic. Various applications can be applied to decide the infection and its stage on the tissues health. The disease diagnosis procedure has been made more efficient by denoising the CT images where the noise is removed. The denoised images encompass a prominent level of elevation in its signal to noise ratio (SNR) values, producing the smoother version of the image for diagnosis function. For developing the quality of the CT images, a variation of methods. The past studies have achieved denoising by utilizing several filters and wavelet denoising methods. Denoising has been achieved. Since they are accomplished of suppressing noise, recent wavelet thresholding [3,4] based denoising methods have proved capable, during the conservation the high-frequency signal details. However, by standard wavelet thresholding methods [5-7], the local space-scale information of the image is not adaptively considered. The threshold [8] at certain scale is a constant for all wavelet coefficients in standard wavelet thresholding based noise reduction methods. In a few applications, wavelet based denoising has been showed efficient. Fundamentally, the noisy image is transformed into the wavelet domain [9], then the wavelet coefficients are shifted to soft or hard thresholding $[10,11]$, and the result has been inverse-transformed in the final step.

\section{METHODOLOGY: PIXEL NORMALIZATION ALGORITHM (PNA) FOR IMAGE DENOISING}

Let us suppose that for a given image represented as I of $\mathrm{m} \times \mathrm{n}$ medians of the mean of the diagonals pixel of neighboring pixel position $\mathrm{P}$, where $\mathrm{f} \in \mathrm{R}^{\mathrm{n}}$ is a noisy image of $n$ pixels; such that I' is optimal denoised images.

Therefore,

$I^{\prime}=\arg \min \left(I-\mathrm{f}_{\mathrm{n}}^{\mathrm{m}}+\sigma \nabla \mathrm{I}_{\mathrm{n}}^{\mathrm{m}}\right)$

Where, $\sigma$ is a scalar which is weighted by the regularization term.

Thus, the neighbor structure of the random pixel position in noisy images is represented as:

If $\mathrm{N}_{\mathrm{b}}$ is the number of nearest neighbor around P. As shown in Fig. 1, we classify the universal set for pixel $\mathrm{P}$ is given by:

$\mathrm{U}=\{\mathrm{N}, \mathrm{W}, \mathrm{S}, \mathrm{E}, \mathrm{NW}, \mathrm{NE}, \mathrm{N} 1, \mathrm{SW}, \mathrm{W} 1, \mathrm{~S} 1, \mathrm{SE}, \mathrm{E} 1\}$

and

$\mathrm{N}_{\mathrm{b}}=\{\{\mathrm{NW}, \mathrm{P}, \mathrm{SE}\},\{\mathrm{NE}, \mathrm{P}, \mathrm{SW}\},\{\mathrm{W}, \mathrm{P}, \mathrm{E}\}\}$

Now, $I_{i, j}$ be the image intensity at the local region of image pixel position 
P. Therefore, diagonal gradient approximation is given by:

$$
\left|\nabla I_{i, j}\right|=\sqrt{\left(I_{i+1, j}-I_{i, j}\right)^{2}+\left(I_{i, j}+1-I_{i, j}\right)^{2}}
$$

If $\nabla \mathrm{I}_{\mathrm{i}, \mathrm{j}}=0$, then either $\mathrm{i}$ or $\mathrm{j}$ falls outside the image of intersection boundaries of the two images; which lead us to classify the images from inbound $\mathrm{Ib}_{\mathrm{o}}=\left\{\mathrm{Ib}_{\mathrm{i}, \mathrm{j}}\right\}$ \& outbound $\mathrm{O}_{\mathrm{b}}=\left\{\mathrm{O}_{\mathrm{Nb}}\right\}$ images at the intersection of the pixel position. Thus, the intersection point of the inbound \& outbound boundaries is represented by $\mathrm{I}_{\mathrm{i}, \mathrm{j}}=\mathrm{O}_{\mathrm{b}}\left(\mathrm{Ib}_{\mathrm{i}, \mathrm{j}}\right)$. Thereby, the weights of intensity normalization at $\mathrm{i}$, j given by $\mathrm{w}_{\mathrm{N}_{\mathrm{b}}}=\left|\mathrm{I}_{\mathrm{i}-1,-1 \mathrm{j}}-\mathrm{I}_{\mathrm{i}-2, \mathrm{j}-2}\right|$ for each outbound pixel position $\mathrm{O}_{\mathrm{Nb}}=\left(\mathrm{Ib}_{\mathrm{i}+1,+1 \mathrm{j}^{\mathrm{j}}}, \mathrm{Ib}_{\mathrm{i}+2,+2 \mathrm{j}}\right)$ connected by the inbound pixel position $[12,13]$. Based on weighted spatial localization of neighboring pixels the denoising problem is more precisely derived as:

$$
\left\|\nabla_{\mathrm{N}_{\mathrm{b}}} \mathrm{I}\right\|_{\mathrm{m}}^{\mathrm{m}}=\sum_{\mathrm{w}_{\mathrm{N}_{\mathrm{b}} \in \mathrm{W}}}\left|\mathrm{w}_{\mathrm{N}_{\mathrm{b}}}\right|^{\mathrm{m}}
$$

PNA

Algorithm:

Input: Noisy Image I

Output: Denoised Image I',

$I^{\prime}=\arg \min \left(I-\mathrm{f}_{\mathrm{n}}^{\mathrm{m}}+\sigma \nabla \mathrm{I}_{\mathrm{n}}^{\mathrm{m}}\right)$

Where, $\sigma$ is a scalar which is weighted by the regularization term and $\mathrm{m}$ and $\mathrm{n}$ belongs to the median values of the diagonal pixels at pixel position $\mathrm{P}$.

Step 1: Begin with $\mathrm{Ib}_{\mathrm{o}}=\{\}$ and $\mathrm{O}_{\mathrm{b}}=\{\}$

Calculate, $\mathrm{N}_{\mathrm{b}}$ is the number of nearest neighbor around $\mathrm{P}$. Then, we classify the universal set for pixel $\mathrm{P}$ is given by:

$\mathrm{U}=\{\mathrm{N}, \mathrm{W}, \mathrm{S}, \mathrm{E}, \mathrm{NW}, \mathrm{NE}, \mathrm{N} 1, \mathrm{SW}, \mathrm{W} 1, \mathrm{~S} 1, \mathrm{SE}, \mathrm{E} 1\}$

and

$\mathrm{N}_{\mathrm{b}}=\{\{\mathrm{NW}, \mathrm{P}, \mathrm{SE}\},\{\mathrm{NE}, \mathrm{P}, \mathrm{SW}\},\{\mathrm{W}, \mathrm{P}, \mathrm{E}\}\}$

Step 2: Add pixel position to $\mathrm{Ib}_{0}$ the inbound pixel to the weight in outbound pixel $\mathrm{O}_{b}$ which form an intersection in $\left(\mathrm{Ib}_{\mathrm{o}}, \mathrm{O}_{\mathrm{b}}\right)$ to the corresponding inbound pixels to $\mathrm{V}$ that are not already in $\mathrm{Ib} \mathrm{b}_{0} . \mathrm{I}_{\mathrm{i}, \mathrm{j}}$ be the image intensity at the local region of image pixel position $\mathrm{P}$. Therefore, diagonal gradient approximation is given by:

$\left|\nabla I_{i, j}\right|=\sqrt{\left(I_{i+1, j}-I_{i, j}\right)^{2}+\left(I_{i, j+1}-I_{i, j}\right)^{2}}$

Step 3: Find median values $(\mathrm{m})$ of from the mean of neighboring diagonal pixels at $\mathrm{P}$.

Step 4: Repeat step 2 and 3 until $\mathrm{Ib}_{\mathrm{o}}$ is homogenous and compute weight of intensity normalization for each pixel position $\mathrm{P}$ given by:

$$
\left\|\nabla_{\mathrm{N}_{\mathrm{b}}} \mathrm{I}_{\mathrm{n}}^{\mathrm{m}}\right\|=\sum_{\mathrm{w}_{N_{b} \in \mathrm{W}}}\left|\mu_{\mathrm{i}}\left(\mathrm{w}_{\mathrm{N}_{\mathrm{b}}}, \mathrm{x}, \mathrm{y}\right) \prod \ldots \prod \mu_{\mathrm{i}+\mathrm{n}}\left(\mathrm{w}_{\mathrm{N}_{\mathrm{b}}}, \mathrm{x}, \mathrm{y}\right)\right|^{\mathrm{m}}
$$

Step 5: A fed forward multi-layered perceptrons (MLP) is used to map the vector valued input $\left\|\nabla_{\mathrm{N}_{\mathrm{b}}} \mathrm{I}_{\mathrm{m}}^{\mathrm{m}}\right\|$ via two hidden layers to vector valued output. Therefore,

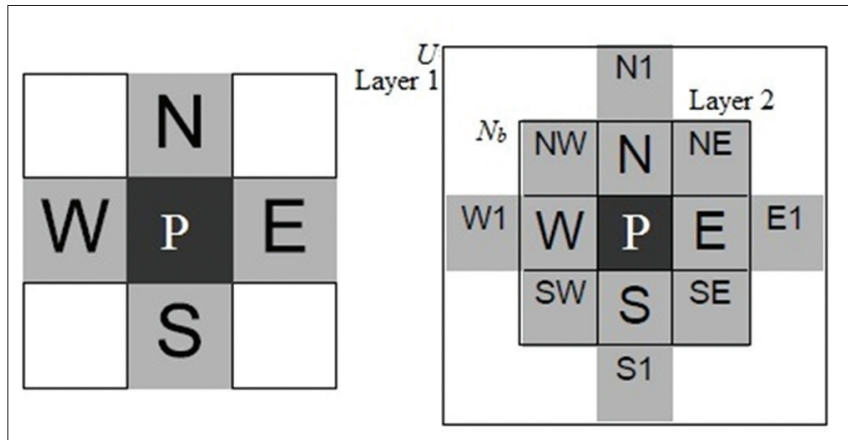

Fig. 1: The neighboring structures of a given pixel in a noisy image

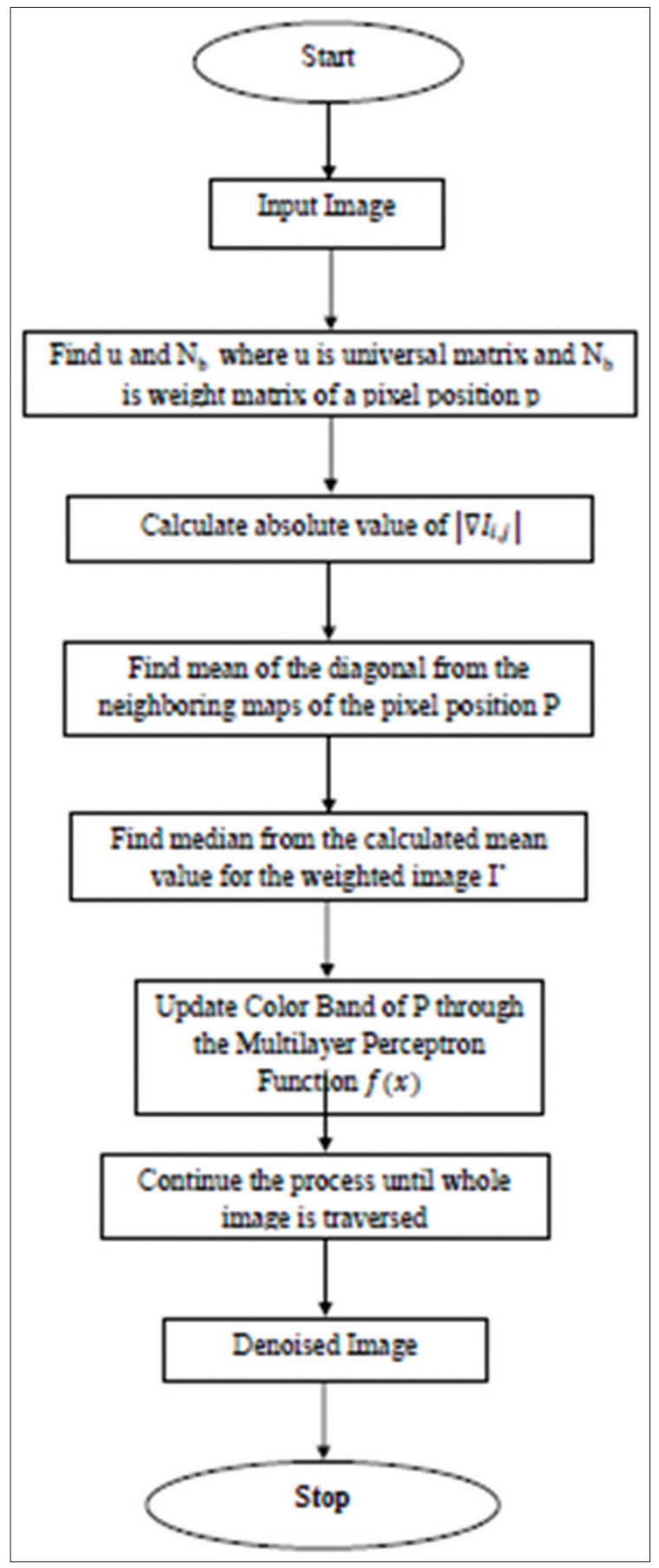

Fig. 2: Flow chart of pixel normalization algorithm 
$f(x)=B_{3}+w_{3} \tanh \left(B_{2}+w_{2} \tanh \left(B_{1}+w_{1} x\right)\right)$

Where the parameterization values is given by $\mathrm{w}_{1}, \mathrm{w}_{2}$ and $\mathrm{w}_{3}$ are the weight matrices. Furthermore, $\mathrm{B}_{1}, \mathrm{~B}_{2}$, and $\mathrm{B}_{3}$ are the vector-valued biases. Since, tanh operates componentwise and the input layer size is 256 ; hence vector of the first hidden layer and the second hidden layer are $\mathrm{SB}_{1}=\tanh \left(\mathrm{B}_{1}+\mathrm{w}_{1} \mathrm{x}\right)$ and $\mathrm{SB}_{2}=\tanh \left(\mathrm{B}_{2}+\mathrm{w}_{2} \mathrm{~V}_{1}\right)$, respectively. Therefore, the architecture of the MLP with two hidden layers is equivalent to 256,200,100.

Step 6: End process.

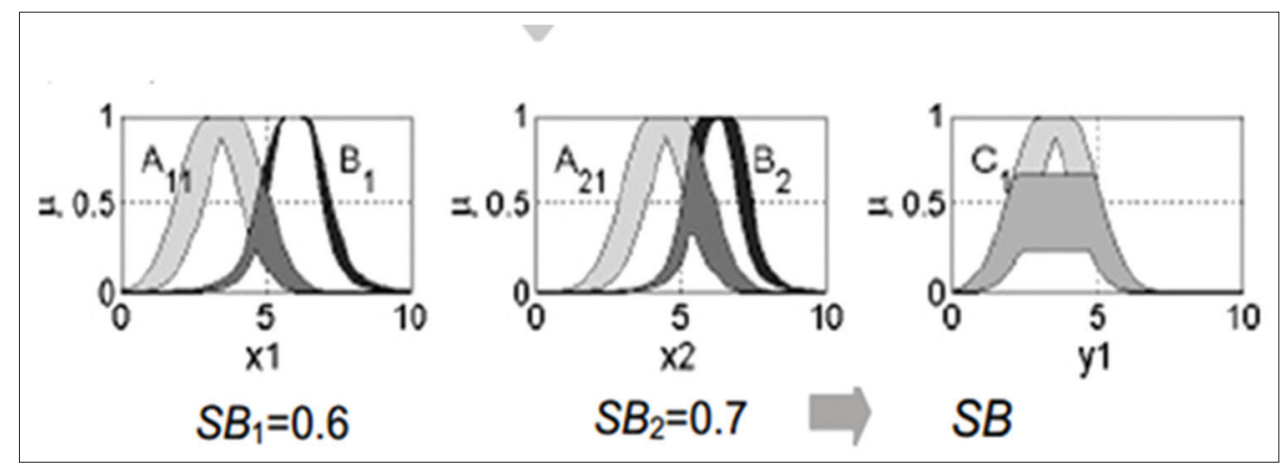

Fig. 3: Membership function for image denoising using neuro-fuzzy type 2 system

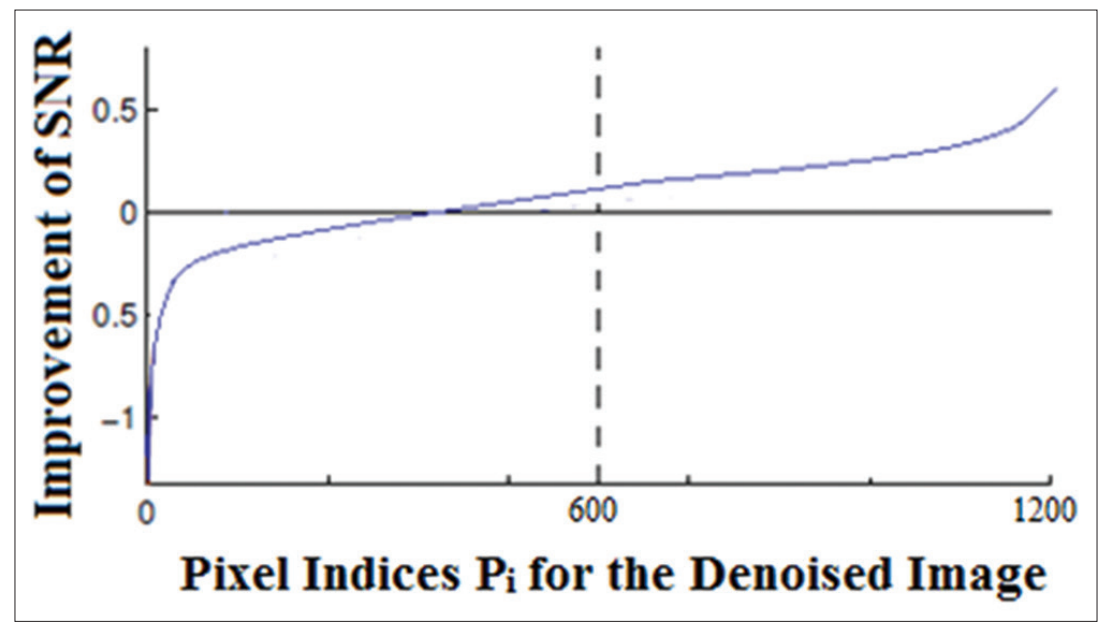

Fig. 4: Profiling of performances of algorithm for 1200 pixel indices $P_{i}$ for the denoised image
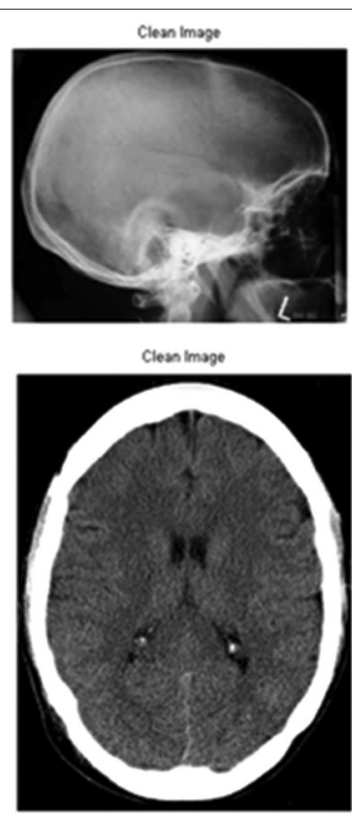

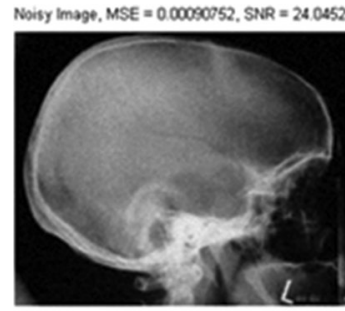

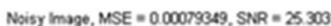

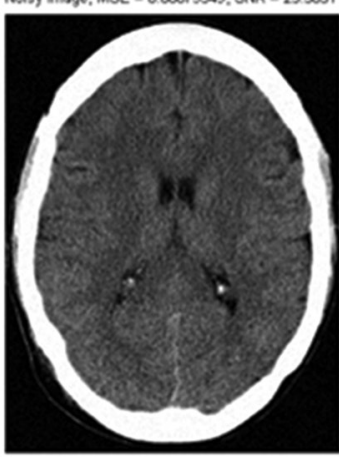

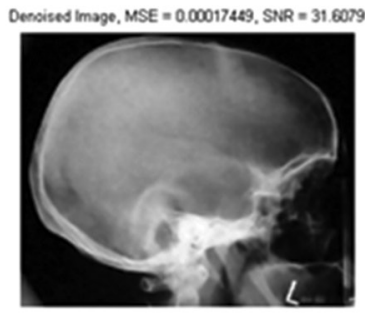

Dencised Image, MSE = 000000053, SNR = 299119

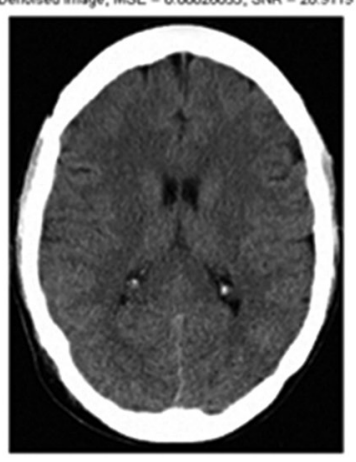

Fig. 5: The sample denoised medical images through the operation of algorithm over noisy images and the corrected denoised output images with its respective mean signal error and signal to noise ratio 


\section{RESULTS AND CONCLUSION}

Data flow diagram of the workflow process is shown in Fig. 2. In this proposed study, the removal of noise from MRIs has been discussed through the novel method of pixel regularization with aid from neurofuzzy type 2 rule sets. As shown in Fig. 5, our study is quite successful in eliminating noise from the essential medical images which shall help in easy detection of cancer or tumors (as shown in above images) for the medical practitioner. The pixel transformation achieved by the proposed method is found to be more efficient than the wavelet methods in image denoising particularly for the removal of Gaussian noise while ensuring that the denoising is done while keeping intact of the integrity and granularity of the image (Figs. 3 and 4). Finally, our results shall help build qualitative and quantitative analysis for further future studies such that the same task attempted through the proposed process has outperformed the wavelet methods; both visually and in terms of mean signal error and SNR.

\section{REFERENCES}

1. Wang Y, Zhou H. Total variation wavelet-based medical image denoising. Int J Biomed Imaging 2006;2006:1-6.

2. Badawi A. Scatterer density in nonlinear diffusion for speckle reduction in ultrasound imaging: The isotropic case. Int J Biol Life Sci 2006;2(3):149-67.

3. Gerig G, Kubler O, Kikinis R, Jolesz FA. Nonlinear anisotropic filtering of MRI data. IEEE Trans Med Imaging 1992;11(1):221-32.

4. Wood JC, Johnson KM. Wavelet packet denoising of magnetic resonance images: Importance of Rician noise at low SNR. Magn Reson Med 1999;41(1):631-5.

5. Manjón JV, Thacker NA, Lull JJ, Garcia-Marti G, Marti-Bonmati L, Robles M. Multicomponent MR image denoising. J Biomed Imaging 2009;2009(18):1-27.

6. Perona P, Malik J. Scale-space and edge detection using anisotropic diffusion. IEEE Trans Pattern Anal Mach Intell 1990;12(7):629-39.

7. Manjon JV, Robles M, Thacker NA. Multispectral MRI de-noising using non-local means. In: Proceedings of MIUA. Aberystwyth; 2007. p. 41-6.

8. de Fontes FP, Barroso GA, Hellier P. Real time ultrasound image denoising. J Real-Time Image Process 2010;1:1-14.

9. Fu S, Ruan Q, Wang W, Li Y. Feature preserving nonlinear diffusion for ultrasonic image denoising and edge enhancement. World Acad Sci Eng Technol 2005;2:148-51.

10. Thaipanich T, Kuo J. An adaptive nonlocal means scheme for medical image denoising. In: Proceedings of SPIE Medical Imaging. Vol. 7623. San Diego, CA, USA, February; 2010

11. Kang SC, Hong SH. A speckle reduction filter using waveletbased methods for medical imaging application. In: Proceedings of $23^{\text {rd }}$ Annual International Conference of the IEEE Engineering in Medicine and Biology Society. Vol. 3. Istanbul, Turkey, October; 2001. p. 2480-3.

12. Rai A. Multispectral image denoising using Bi-directional recurrent neural network with DPCA algorithm. J Image Process Pattern Recognit Prog 2015;2(1):25-30.

13. Rai A. A novel decomposable pixel component analysis algorithm for automating multispectral satellite image denoising. Res Rev J Embed Syst Appl 2015;2(3):18-25 